\title{
A Smart Gateway Architecture for Improving Efficiency of Home Network Applications
}

\author{
Fei Ding, ${ }^{1,2}$ Aiguo Song, ${ }^{3}$ En Tong, ${ }^{1,2}$ and Jianqing $\mathrm{Li}^{3}$ \\ ${ }^{1}$ School of Information Science and Engineering, Southeast University, Nanjing 210096, China \\ ${ }^{2}$ R\&D Center, China Mobile Group Jiangsu Co., Ltd., Nanjing 210029, China \\ ${ }^{3}$ School of Instrument Science and Engineering, Southeast University, 2 Sipailou, Nanjing, Jiangsu 210096, China
}

Correspondence should be addressed to Aiguo Song; a.g.song@seu.edu.cn

Received 26 September 2015; Accepted 29 November 2015

Academic Editor: Gwanggil Jeon

Copyright (C) 2016 Fei Ding et al. This is an open access article distributed under the Creative Commons Attribution License, which permits unrestricted use, distribution, and reproduction in any medium, provided the original work is properly cited.

\begin{abstract}
A smart home gateway plays an important role in the Internet of Things (IoT) system that takes responsibility for the connection between the network layer and the ubiquitous sensor network (USN) layer. Even though the home network application is developing rapidly, researches on the home gateway based open development architecture are less. This makes it difficult to extend the home network to support new applications, share service, and interoperate with other home network systems. An integrated access gateway (IAGW) is proposed in this paper which upward connects with the operator machine-to-machine platform (M2M P/F). In this home network scheme, the gateway provides standard interfaces for supporting various applications in home environments, ranging from on-site configuration to node and service access. In addition, communication management ability is also provided by M2M P/F. A testbed of a simple home network application system that includes the IAGW prototype is created to test its user interaction capabilities. Experimental results show that the proposed gateway provides significant flexibility for users to configure and deploy a home automation network; it can be applied to other monitoring areas and simultaneously supports a multi-ubiquitous sensor network.
\end{abstract}

\section{Introduction}

The IoT (Internet of Things) is considered one of the major communication advances in recent years, since it offers the basis for the development of independent cooperative services and applications [1]. Extensive research is underway to explore the potential applications using this concept in different areas, such as a ZigBee-based, wearable scheme for monitoring physiological parameters [2], monitoring animal presence and pasture time over an extended area [3], the management of medication and health care [4], and, in particular, home network applications (HNAs). The IoT potential for HNAs is reported in [5-8]. The implementation of IoT for monitoring the environmental conditions of a home is proposed in [5]. A smart home testbed based on the pedagogical model of project-based learning (PBL) for undergraduate education is proposed in [6]. A wireless home automation network for indoor surveillance is presented in [7]. A synchronous smart and wireless home energy management scenario was proposed in [8] and [9], respectively. Each of these studies identifies potential capacities for self-configuration, comprehensive management, and communication capabilities.

For HNAs, the home gateway is the most important sink node (SN) that provides access and management capabilities for the child nodes (CNs), such as home devices or smart home terminals. Many articles about ZigBee-based home gateways have been published recently [10-12]. Authors in [10] propose a ZigBee-based homecare gateway that deals with the collection of terminal medical data and eventually passes the data to the doctor for remote diagnosis. Authors in [11] introduce a household controller supporting both ZigBee and infrared communication. An OSGi-based architecture for the dynamic integration of ZigBee home networks into home gateways is proposed in [12], where ZigBee home network devices are represented as device proxy service bundles. However, according to these HNA scenarios, data interaction between $\mathrm{SN}$ and $\mathrm{CN}$ is achieved mostly by proprietary 


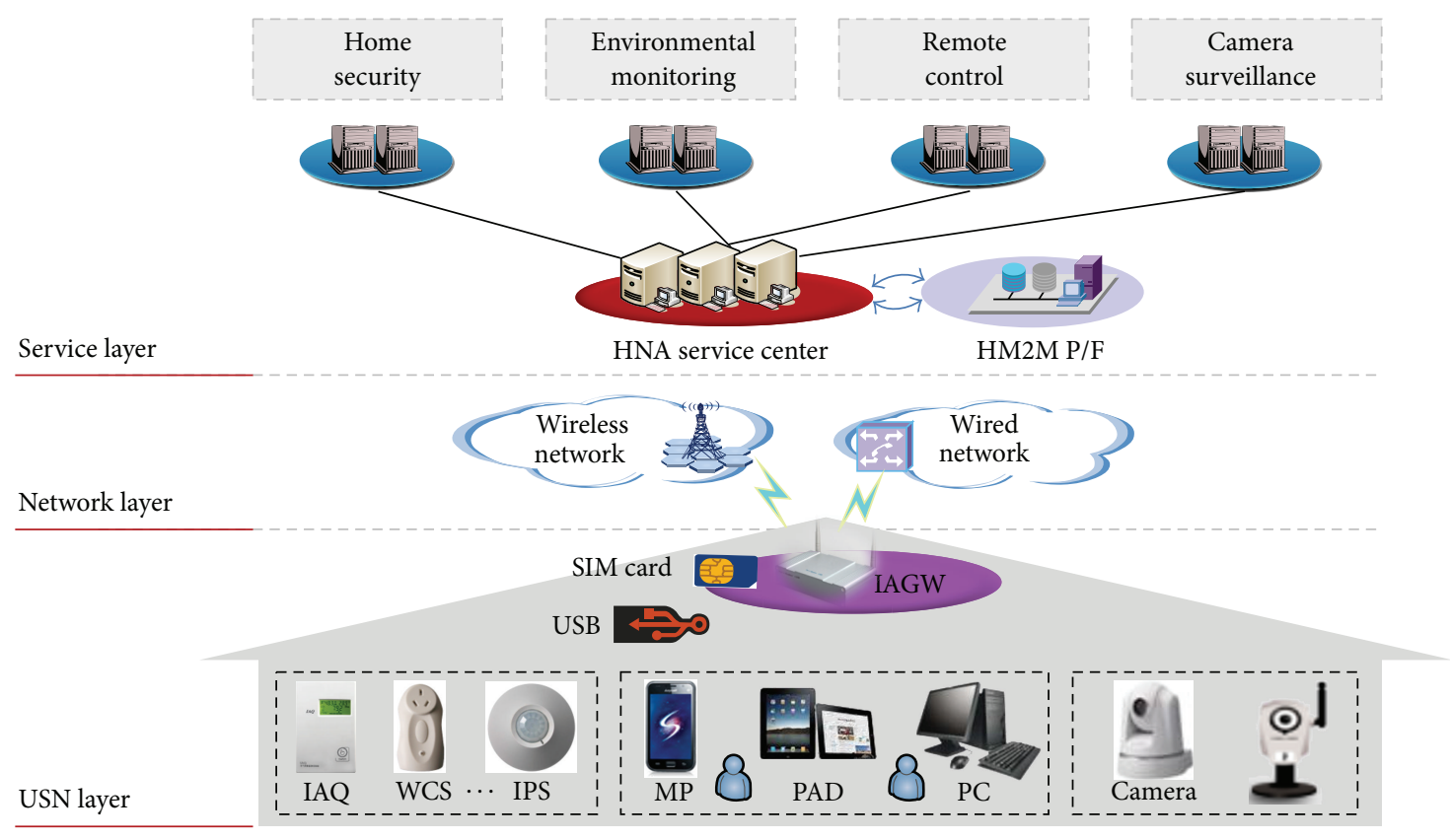

Figure 1: A M2M platform based home network architecture.

wireless protocol, and both $\mathrm{SN}$ and $\mathrm{CN}$ are specially designed for an HNA scheme, and, as such, lack interoperability. These proprietary gateways and their systems are difficult to extend so as to support new services, integrate new data, or interoperate with other HNAs.

Machine-to-machine platform (M2M P/F) integrated service schemes which offer self-configuration and expandability have significant potential for improving the efficiency of traditional home automation systems [13]. In [14], a snapshot is proposed of the latest progress in M2M standardization of things such as requirements, architecture, candidate protocols, security aspects, and device management. In [15], an IoT-based retractable bollard management system is described to govern vehicular access appropriated for restricted urban areas. In [16], biomedical applications and various low power wireless sensor and link technologies are discussed, but the scheme integration proposed is only suited to the BlackBerry system. In [17], M2M architecture for applications in healthcare, energy management, and entertainment is proposed, and the performance tradeoffs inherent in existing designs are evaluated.

In this paper, an integrated access gateway (IAGW) architecture is proposed with standard interfaces for supporting various application nodes in home environments, ranging from on-site configuration to node and service access. Both the upward and downward communication interfaces of the IAGW are standardized. The IAGW based home network system architecture adopts a separation principle between terminal management and service implementation; the former is realized by the $\mathrm{M} 2 \mathrm{M} \mathrm{P} / \mathrm{F}$, and the latter is provided by the home service layer. The working mode and parameter configuration of the IAGW gateway can be set using a web console. If the configuration of the IAGW is completed correctly, it will allow management by the M2M
$\mathrm{P} / \mathrm{F}$ automatically. Meanwhile, the system service data are directly transferred to the home service layer through the IAGW. Based on the proposed IAGW and the M2M P/F fusion architecture, the home sensors and services can be standardized and easily managed. Thus, it can reduce the redevelopment workload and promote the interoperability between the multi-HNA systems. The system architecture, the design methods of the IAGW based HNA, and the demonstration experiment will be discussed in Sections 2-4.

\section{System Overview}

As shown in Figure 1, the hierarchical architecture of the home network system consists of 3 layers: the ubiquitous sensor networks (USN) layer, the network layer and the service layer.

The main role of the IAGW is data transmission between the CNs and the network layer. The CNs in the USN layer include indoor air quality (IAQ), wireless control socket (WCS), infrared proximity sensor (IPS), video camera, and other sensors or actuators in the HNA. Except for the video camera, the other CNs are integrated with ZigBee modules and network ability. The video camera connects to the IAGW gateway through a Wi-Fi wireless interface or an RJ45 wired interface. Users enjoy the convenience and safety of HNA services through the personal computer (PC), mobile phone (MP), and PAD. The network layer consists of a wired network and a $3 \mathrm{G} / 4 \mathrm{G}$ wireless cellular network. The service layer includes an HNA service center (HNASC) and a home M2M platform (HM2M P/F); the former is responsible for home services management, while the latter provides interfaces to the IAGW and supports gateway management. The HNASC supports receiving, authenticating, and storing realtime data collected by the IAGW. It also connects with the 


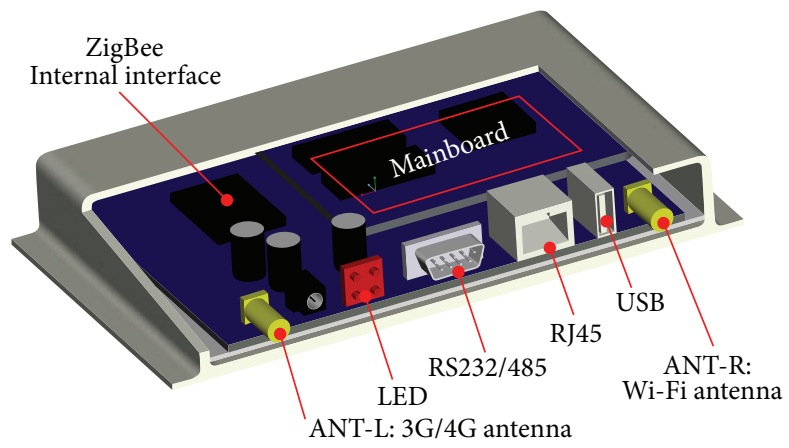

(a)

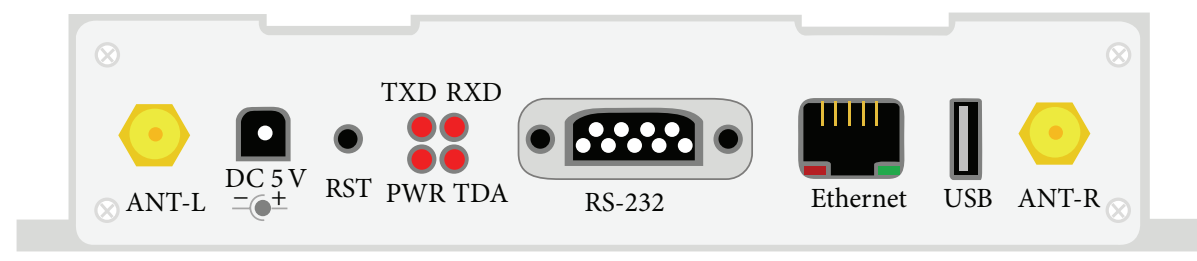

(b)

FiguRE 2: Hardware architecture of the proposed gateway. (a) Stack structure of the proposed gateway. (b) Front view of the gateway interface side.

business operating support system (BOSS) to realize operations like business order synchronization and push information to users in the form of hand-held terminal and short message service (SMS).

\section{Design Methods}

3.1. IAGW Prototype. Figure 2 shows the stack structure hardware architecture of the proposed IAGW. The IAGW prototype is designed to provide standard access to the CNs deployed in the HNA system. The mainboard is the core unit of the IAGW that is designed with a MCU, a SDRAM, and a NAND flash; it runs Linux 2.6 or a later operating system (OS). The USB interface is used to connect a memory card for local data storage, especially video monitoring data. The 4 LED indicators of the IAGW represent different working states, corresponding to power on, network connect/disconnect, data transmission, and alarm on/off, respectively.

The IAGW proposes an onboard 22-pin internal interface and an RS232/485 external interface connected to the mainboard, which are convenient for ZigBee SN integration. If user needs to connect and control other wireless standard home devices, the corresponding SN can be redeveloped and integrated through the two interfaces. The dimensions of the onboard internal interface that adjusts to the ZigBee SN are shown in Figure 3.

3.2. Functional Architecture. The functional architecture of the IAGW is shown in Figure 4. The USN layer interface is realized through the ZigBee radio and its networking ability. The public network interface mandates an IP-based network, such as $2 \mathrm{G} / 3 \mathrm{G} / 4 \mathrm{G}$, or Ethernet. The USN Manager
Dimensions of customer interface (unit: $\mathrm{mm}$ )

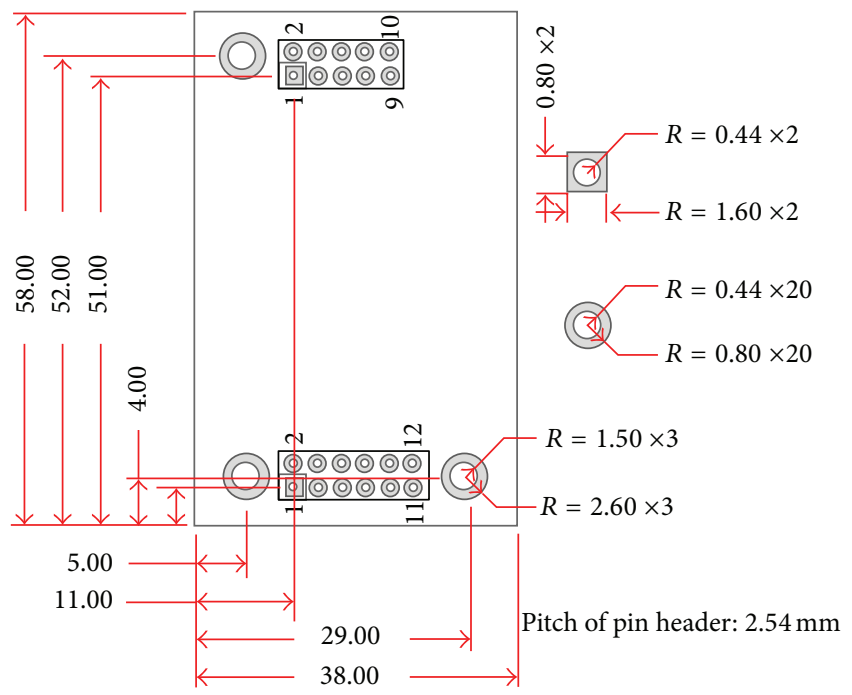

FIGURE 3: Dimensions of the onboard internal interface (unit: $\mathrm{mm}$ ).

module manages the home USN, which consists of providing a uniform network interface to the packet manager or other modules, detection of USN network status (connection status OK/NG), and acquisition of all of the USN network service types through the USN I/F.

The "Registration" module is used to manage node registration to the IAGW and request gateway registration to the HM2M P/F. Meanwhile, the "Gateway Configuration" module provides the communication parameters and working mode configuration of the IAGW through HM2M P/F. 


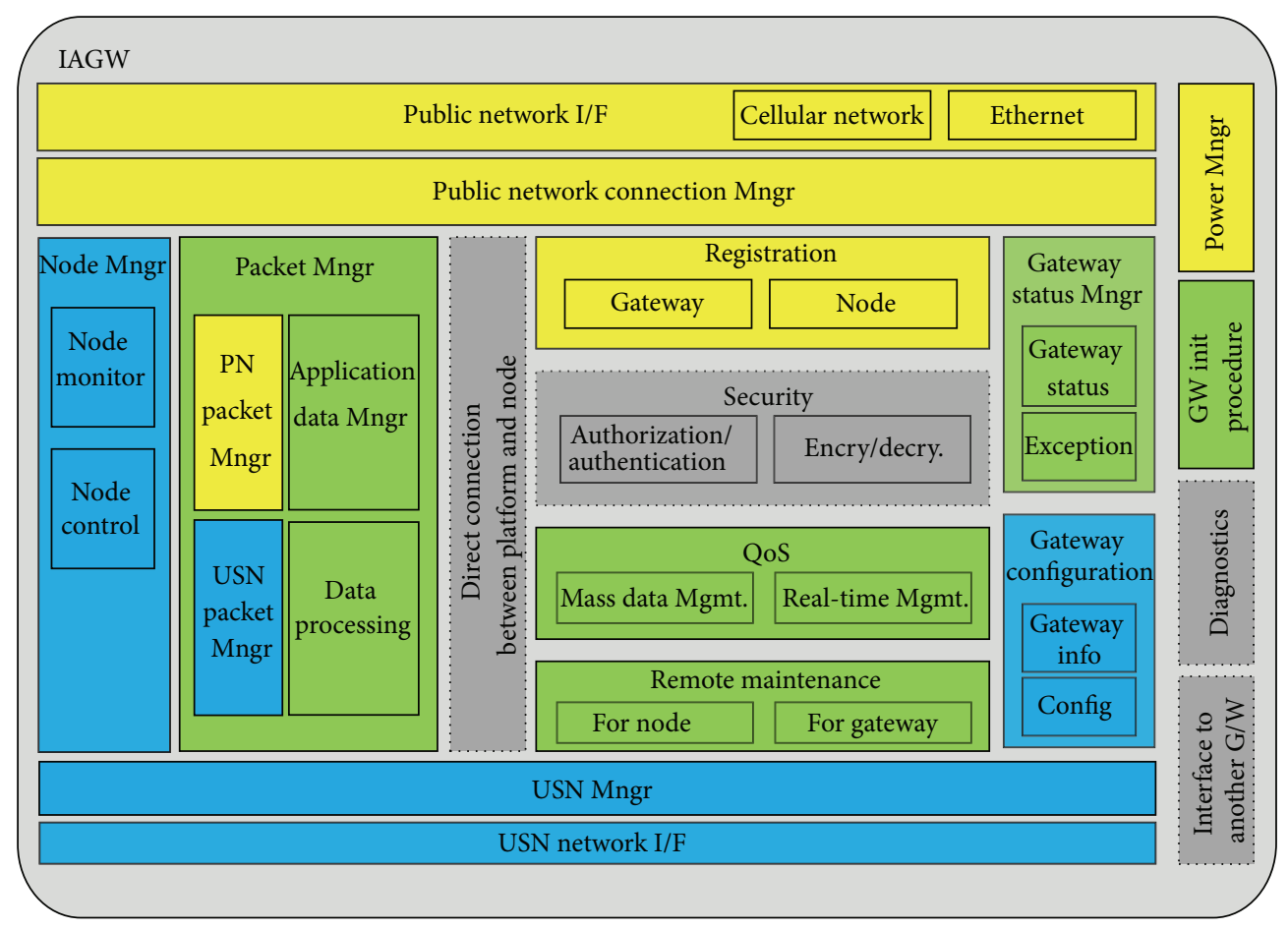

FIGURE 4: Functional architecture of the proposed IAGW gateway.

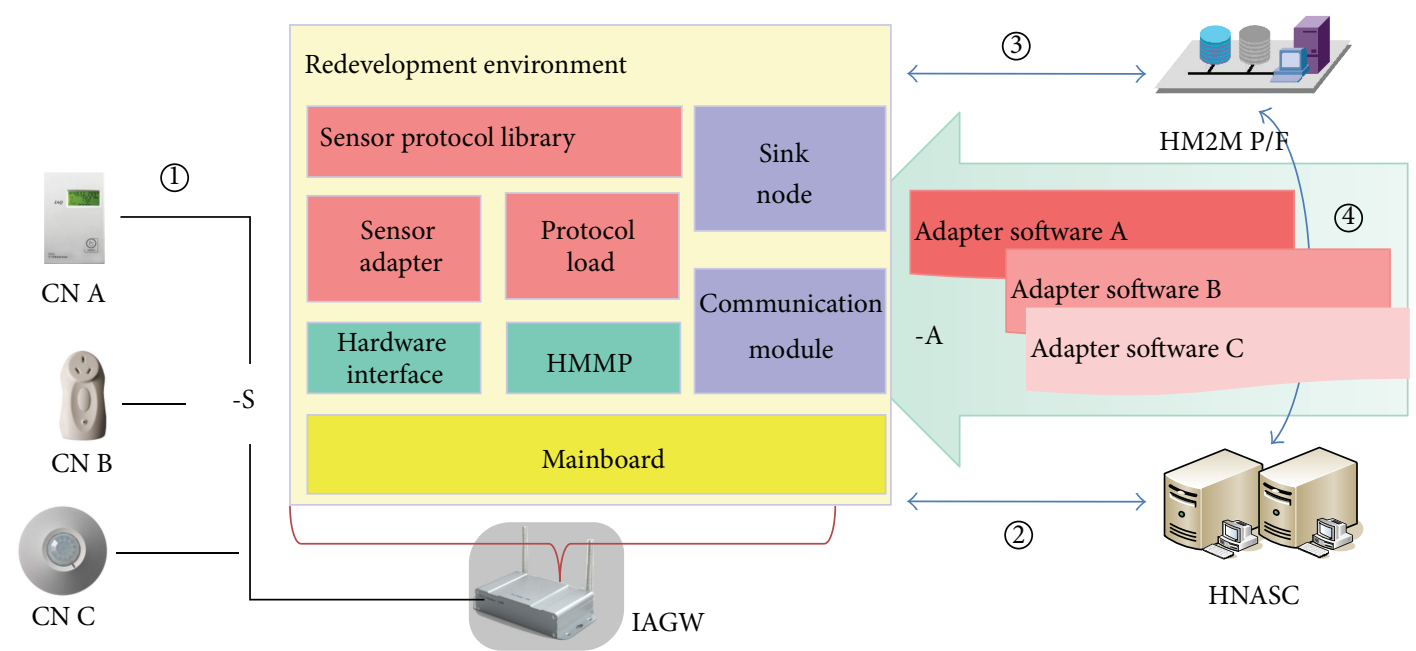

FIGURE 5: Interaction architecture of the IAGW based home network application.

The "Node Mngr" module manages CNs, including aspects such as node ID management, node status monitoring, and node control. The node monitor is a submodule of the node manager that monitors the node's status. The "Packet Mngr" module of the proposed IAGW repacketizes data received from CNs to a standard form and sends them to HNASC $\mathrm{P} / \mathrm{F}$, parses requests initiated by the platform, and packetizes them into the appropriate form to send to the CNs. The "Security" module includes authorization/authentication and en/decryption, and the "QoS (Quality of Service)" module guarantees service quality. The "Remote Maintenance" module supports remote maintenance, such as IAGW and SN software upgrades from the HM2M P/F.

3.3. Adaptive Access and M2M Business Interaction Method. As shown in Figure 5, the IAGW based HNA system can be divided into 2 kinds of communication interfaces: the HMMP- (Home M2M protocol-) S and HMMP-A.

The HMMP-S interface (step (1)) realizes a ZigBee-based wireless communication standard protocol between the CNs 


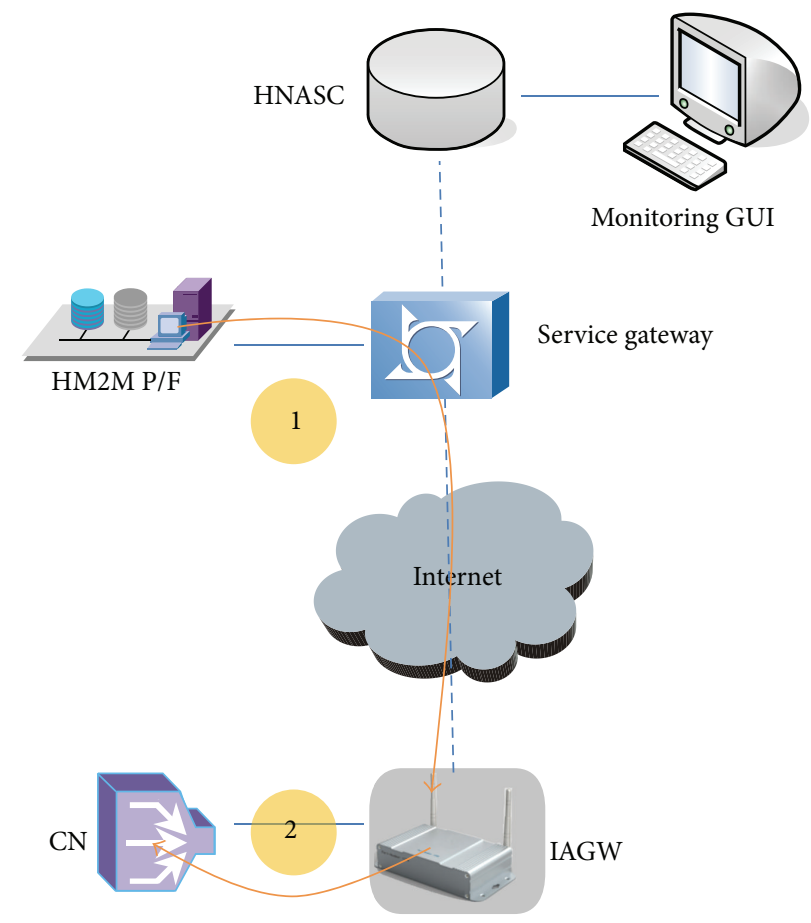

FIGURE 6: Configuration procedure of the home terminal.

and the IAGW, so as to solve the access of the various types of home terminals. It relates to home network attributes, reading the CNs' ID and description information and setting the communication port of the ZigBee SN. It relates to home network services, acquiring all of the home network service types through the IAGW. The HMMP-A protocol (steps (2), (3), and (4)) belongs to the data transmission interface between the IAGW and the HM2M P/F. The HMMP-S interface protocol is forwarded directly to the HMMP-A interface via the IAGW, and HMMP-A protocol includes the packet of the HMMP-S. Meanwhile, if the IAGW connects to the HNASC platform, the HMMP-A protocol should be the expansion of the HMMP-S protocol, so that the reliability of communication between the IAGW and the HM2M P/F, $\mathrm{HM} 2 \mathrm{M}$ P/F, and HNASC can be secured.

The HM2M P/F uses CONFIG_SET command to configure the various home terminals. As shown in Figure 6, the adapter software according to a home terminal transmits through the HM2M P/F (step 1) and remote push to the IAGW (step 2).

Aiming at $\mathrm{CN}$ node management based on HMMP-S protocol, the IAGW takes appropriate action based on the status, such as adjusting the data sending rate or the error status. Meanwhile, it reports node status to the HM2M P/F through the IAGW, allowing to manage the status regularly. HMMP-S protocol also implements control of the CNs' working behaviors, and node control commands are initially issued by the IAGW or HNASC P/F. Home node data acquisition and processing flow are shown in Figure 7: step 0 represents the configuration of the home $\mathrm{CN}$, including data acquisition and data reporting rules; step 1 represents the home node using sensor adapter protocol, which is loaded by the HM2M P/F to read data; step 2 indicates that the home node uses TRANSPARENT_DATA command to transmit data to the HNASC P/F; step 3 presents the hand-held monitoring and displays $\mathrm{CN}$ sensing data and status.

3.4. User Development Procedure. Figure 8 shows the user development step and interface in the proposed HM2M P/F based home network system. By configuring the communication port of the IAGW, the communication between the IAGW and $\mathrm{SN}$ is secured. After port configuration is finished, it would be autosaved to the internal memory of the IAGW.

If the HMMP-S communication configuration is finished, then the communication parameters of the HMMP-A can be set. The main parameters that should be configured include the IP address, the port, and the secret keys of the HNASC $\mathrm{P} / \mathrm{F}$. The IAGW also can be configured in encryption mode. When the COMM_CONFIG data are executed, the IAGW sends a communication key request to the HM2M P/F, which is automatically assigned by the latter. Between the CNs and the IAGW, also the IAGW and the M2M P/F, monitoring and maintenance of communication are implemented with HEART_BEAT and HEART_BEAT_ACK packets. Hence, the data transmission link of the HNA system has been established; the HM2M P/F can implement remote management for the IAGW and CNs, and through the HNASC P/F to support kinds of home services for user.

\section{System Implementation}

4.1. Prototype Gateway Based HNA. As shown in Figure 9, a testbed of a home network system for validating the basic functions of the proposed smart IAGW has been created. The 

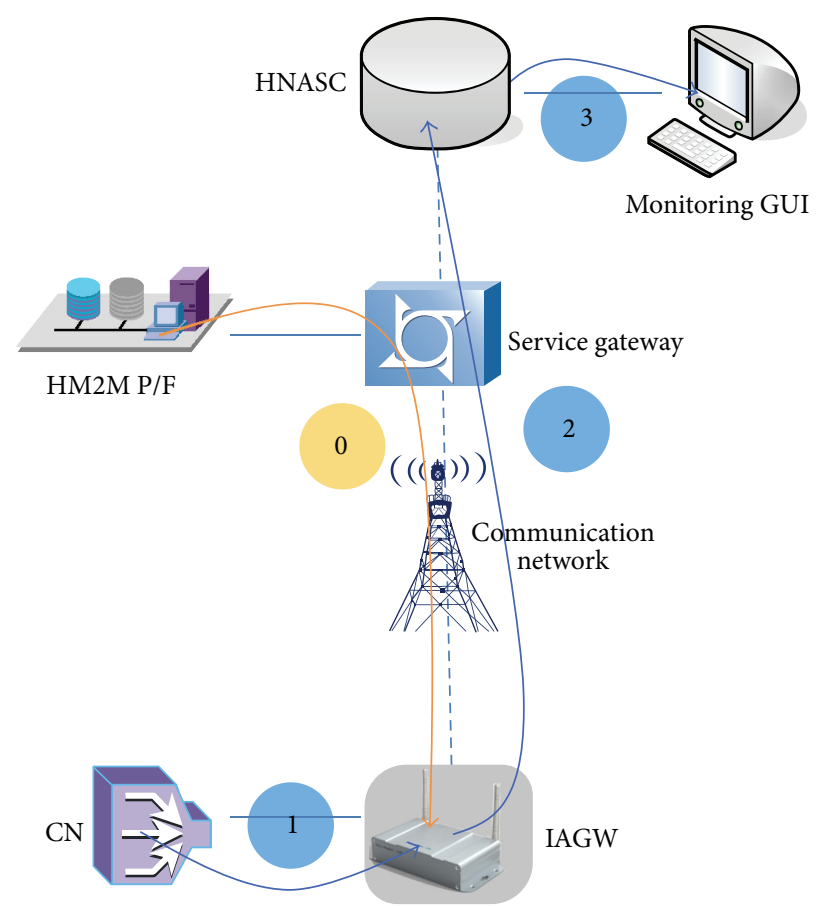

Figure 7: Data acquisition and processing flow of the home node.

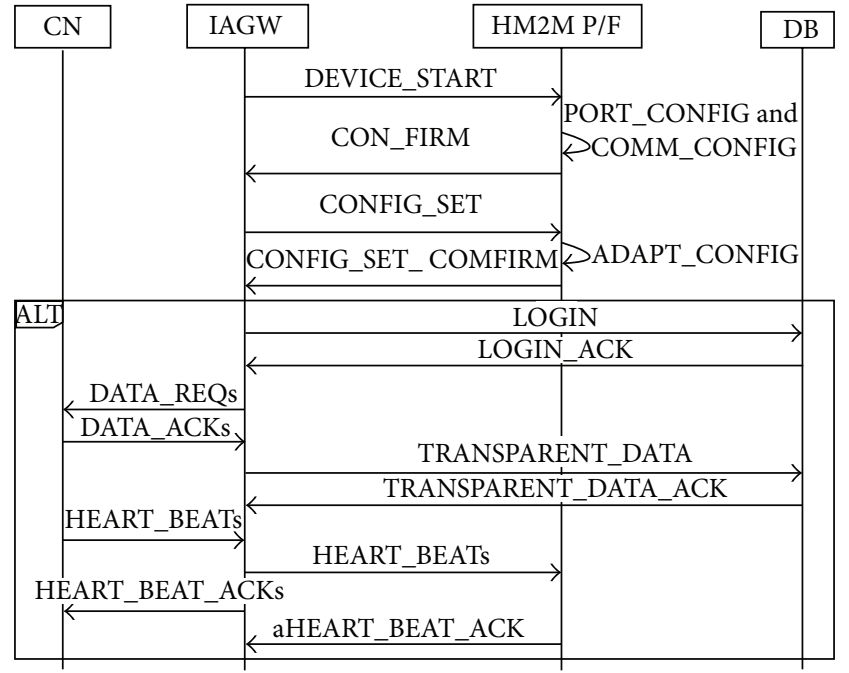

Figure 8: Control flow of the IAGW based M2M system.

testbed includes an IAGW (SN), two IAQ nodes, a WCS node, a smoke detector, a passive infrared (PIR) node installed in the doorway, and an alarm node, and the external antenna of the ZigBee radio is installed in the Wi-Fi antenna interface (as shown in Figure 2), while the Wi-Fi module used an onboard antenna. The comparison between the traditional gateway and the IAGW is listed in Table 1 . The IAGW and the $\mathrm{CN}$ nodes communicate with each other by a ZigBee-based mesh network. A monitoring camera communicates with the IAGW and two types of user service clients (consisting of a local client and a remote hand-held client) through Wi-Fi wireless technology.

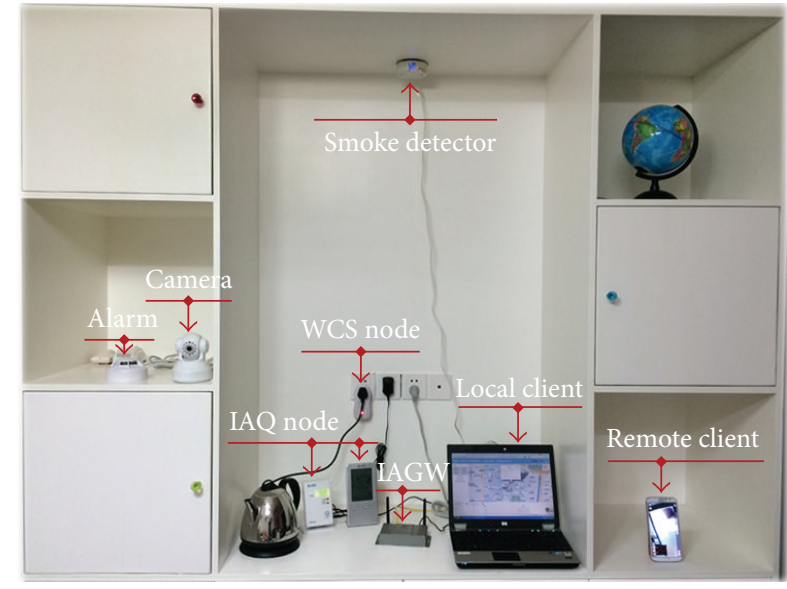

Figure 9: Testbed setup for the demonstration experiments.

The IAGW starts up after connecting to PC using RJ-45 cable, and its working parameters are configured by a web browser, as shown in Figure 10, and after its configuration is complete, it will reboot automatically in response to the parameter configuration. The connection configuration graphic user interface (GUI) of the IAGW is shown in Figure 10(a). In the platform selection suboption, the IAGW chooses to connect to the HM2M P/F. In the basic info suboption, it configures the IP address, port number, and transmission protocol of the HNASC P/F. In the registration mode suboption, it inputs the IAGW serial number and the base, up and down passwords. As shown in Figure 10(b), the communication parameters between the mainboard of the 


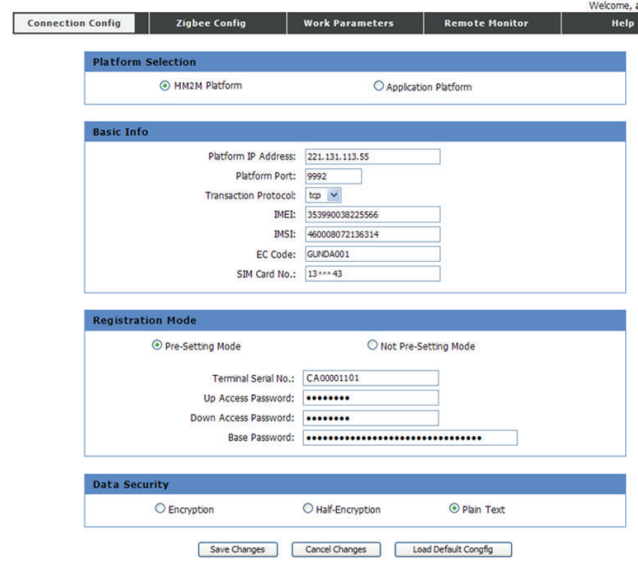

(a)

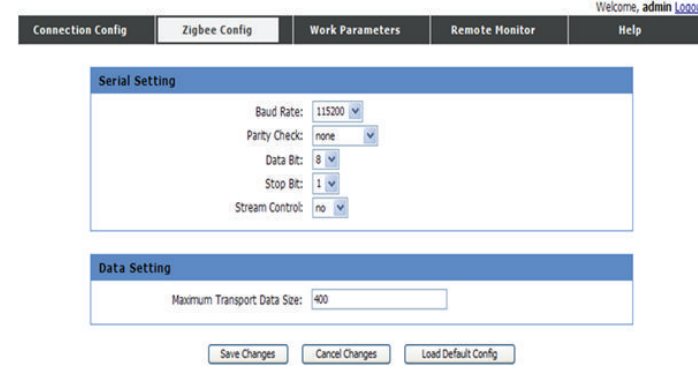

(b)

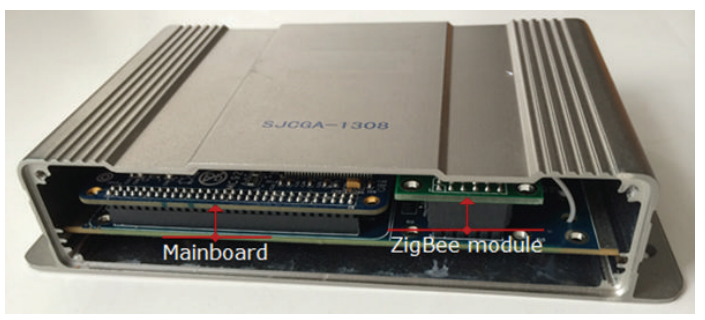

(c)

FIgURe 10: Parameters setting GUIs of the prototype IAGW with the web console. (a) Connection configuration GUI. (b) ZigBee communication configuration GUI. (c) Side view of the proposed prototype gateway.

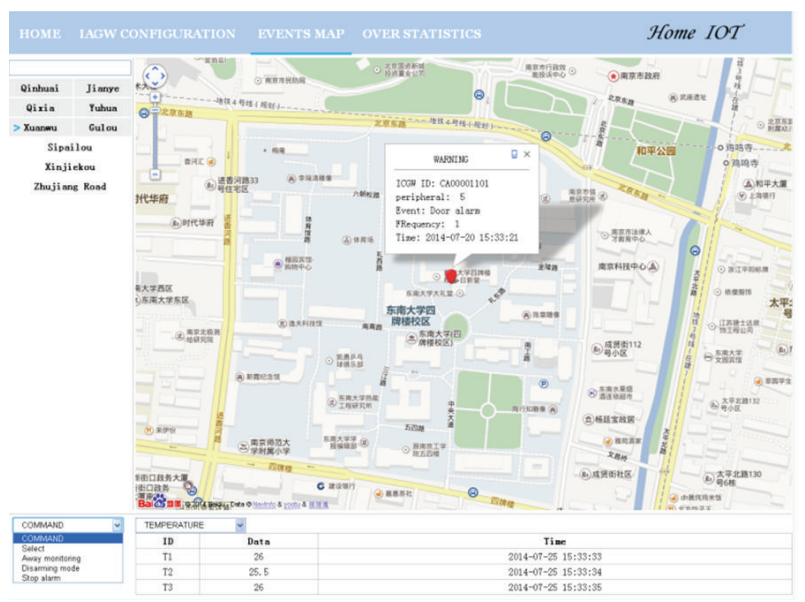

FIGURE 11: Digital map navigation GUI for monitoring platform.

IAGW and the ZigBee SN are implemented according to the ZigBee configuration options.

Figure 11 shows the local monitoring client of the home network system. The GUI functions include data acquisition and management, graphic displays, person-machine dialogue, and real-time and historical database storage. User can control every device in the home network conveniently by using this service client. Three standard monitoring scenes for the home network system, including away monitoring, disarming mode, and a stop alarm, are set for choosing. User can conveniently select the working mode of the system. Once an alarm occurs, it will automatically occur in the PC client and locate the alarm event in the event map GUI. The hand-held remote client can receive the alarm SMS notification; it also has remote access to the home camera, making it convenient for the user to confirm the alarm.

User can remotely check the home environment parameters in real-time and access live video through the hand-held MPs. The GUI running on the user MPs consists of 5 modules, including warning information, node management, realtime data, service instruction, and system configuration. As shown in Figure 12, (a) is the indoor temperature monitoring chart and (b) is the monitoring video of the proposed testbed. The former uses different colors to distinguish the temperature alarm interval, which makes it convenient for user to understand whether it exceeds a set threshold. The latter uses the Wi-Fi network attached to the user's MPs to acquire real-time monitoring video and ensure home safety.

4.2. Demonstration Experiment. Some tests are carried out to evaluate the stability of our IAGW based HNA system. The IAQ node in the proposed testbed senses the home environment in real time; the temperature, humidity, and $\mathrm{CO}_{2}$ data are collected and sent to the HNASC P/F through the IAGW at intervals of 5 seconds. The tests last for 24 hours, and the data are stored and visualized at the HNASC P/F in real time. The sample results of the home environmental monitoring experiments are shown in Figure 13. At 17:10 and 4:10, the large curve fluctuations are caused by blowing air to 


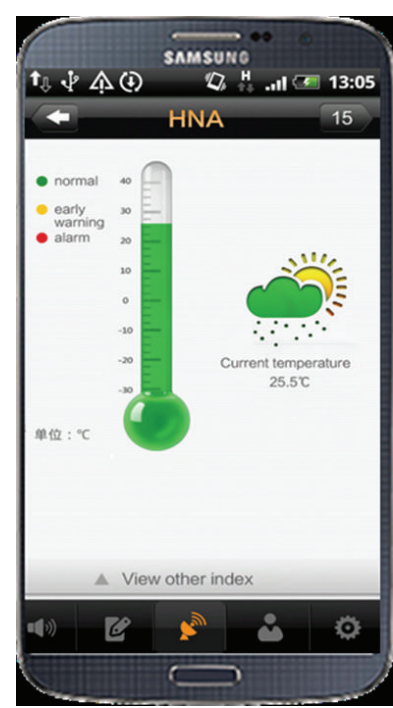

(a)

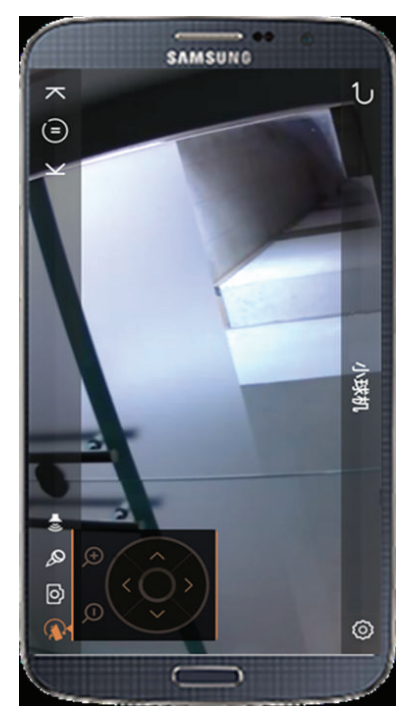

(b)

FIGURE 12: Graphic user interfaces running on hand-held terminals. (a) Indoor temperature GUI. (b) Remote video access GUI.

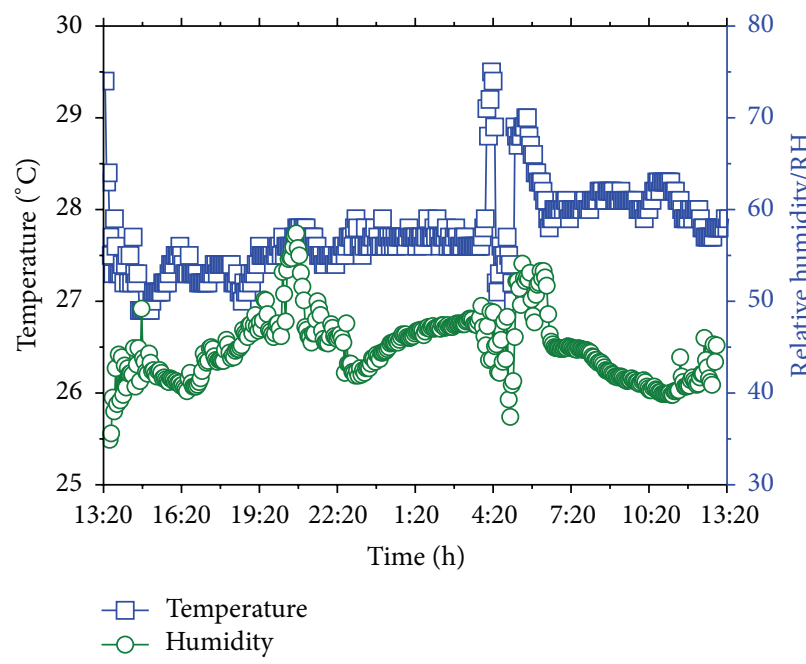

(a)

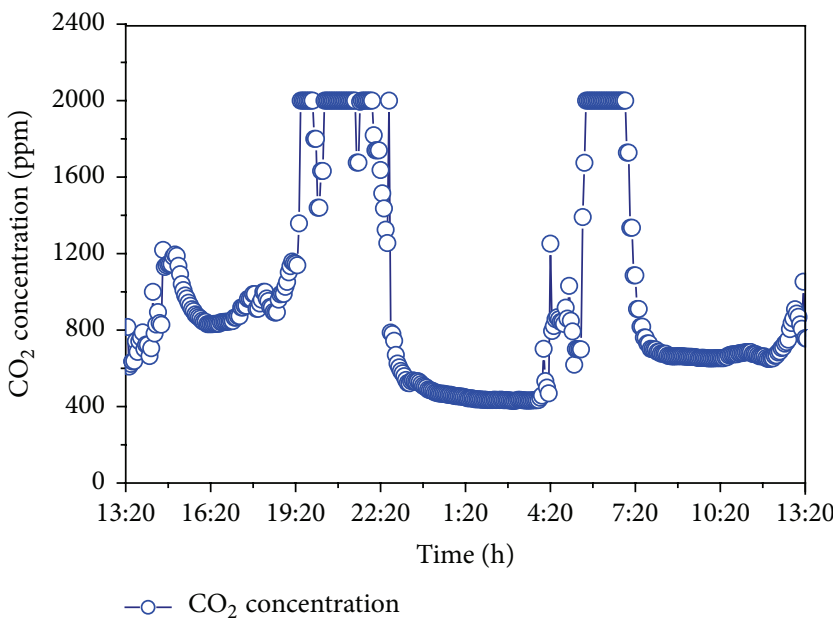

(b)

FIGURE 13: Sample results of the indoor environmental monitoring experiments in 24 hours: (a) temperature and humidity and (b) $\mathrm{CO}_{2}$.

TABLE 1: The comparison between the traditional gateway and the IAGW.

\begin{tabular}{|c|c|c|c|}
\hline ID & Characteristic & Conventional gateways $[2,3,8-10]$ & IAGW \\
\hline 1 & Multi-interface & $\begin{array}{l}\text { Mainly support ZigBee radio and have } \\
\text { poor expansion }\end{array}$ & $\begin{array}{l}\text { Using the stack hardware structure, it } \\
\text { supports ZigBee, Wi-Fi, and Bluetooth } \\
\text { radio and is easy to expand }\end{array}$ \\
\hline 2 & Secondary development ability & Need a large amount of work & $\begin{array}{l}\text { New development workload is relatively } \\
\text { small }\end{array}$ \\
\hline 3 & Communication link monitoring & Need to add custom development & $\begin{array}{c}\text { Automatic support after system } \\
\text { development }\end{array}$ \\
\hline 4 & System openness & $\begin{array}{l}\text { Based on terminal development, all } \\
\text { interfaces are private }\end{array}$ & $\begin{array}{l}\text { The interfaces are standard and the } \\
\text { openness and source utilization of the } \\
\text { system are enhanced }\end{array}$ \\
\hline
\end{tabular}




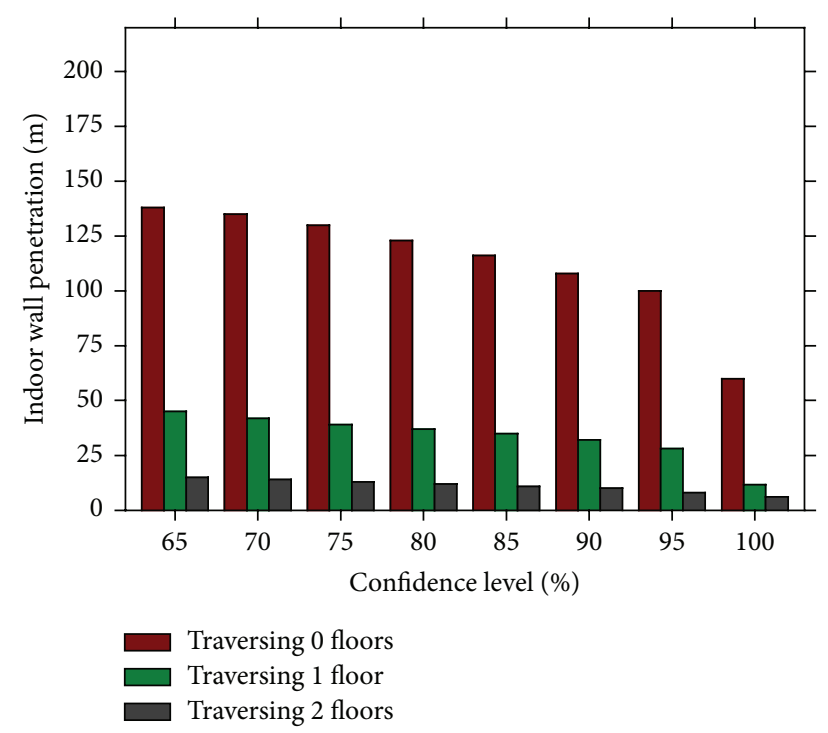

FIGURE 14: Node-to-node spacing capability.

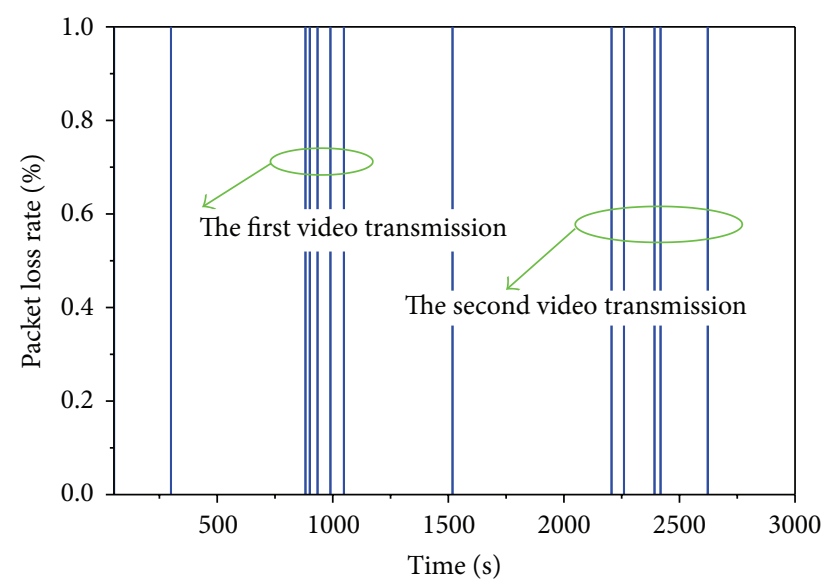

FIGURE 15: Performance evaluation between the two same frequency technologies.

an IAQ node, and the room temperature, humidity, and $\mathrm{CO}_{2}$ changes during one day are displayed in these figures.

Figure 14 plots node-to-node spacing versus confidence level for situations where there is no directional antenna and no line-of-sight present. The testing method is carried out by continuously broadcasting the control data and receiving the successfully returned signal via the ZigBee network. It is certain that when a link is set up inside a building, it will exhibit a shorter range than that suggested by the free-space guidelines, even when a line-of-sight is present. In cluttered environments, the range reduction can be very significant. In fact, radio waves will propagate through brick walls, concrete floors, and plasterboard partitions, among other materials, but a loss will be incurred (as compared with free-space propagation).

The IAGW supports both ZigBee and Wi-Fi $2.4 \mathrm{GHz}$ band wireless standards. In our proposed HNA system, the demand for ZigBee-based data transmission is much larger

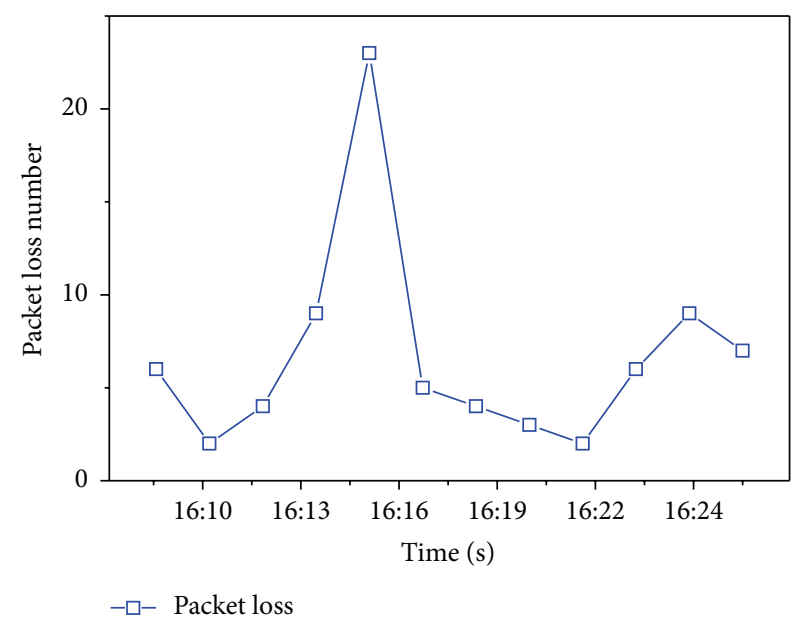

FIgURE 16: The packet loss monitoring based on the HM2M P/F.

than that for Wi-Fi communication. The influence of Wi-Fibased video transmission on ZigBee wireless communication is shown in Figure 15. When the home network system only uses ZigBee wireless data transmission, the system packet loss rate is about $0.4 \%$; when the user starts the remote video monitoring task, the system packet loss rate reaches about $7.6 \%$. Hence, in the HNA system, the user needs to balance video access with system data packet loss. The optimal working mode of the HNA system is to minimize the number of video access services unless an alarm occurs. This can improve system reliability effectively.

Based on the HM2M P/F, the remote data interaction between the IAGW and the USN layer can be monitored in real time. Figure 16 indicates the periodically monitoring results of packet loss in a live cellular network, which helps to evaluate the wireless communication quality and emergency response. In poor wireless network condition, the failed transmitted test data of the USN layer will be cached in the IAGW integrated storage and will be submitted to the HNASC in the next data transmission process with the normal network link working stage.

\section{Conclusions}

A smart gateway architecture for improving the efficiency of home network applications is proposed in this paper. The gateway has stack architecture and provides on-board standard internal and external interfaces which make wireless communication module integration convenient. Among the gateway and various home sensor nodes, as well as between the gateway and the service layer, the gateway realizes the interface standard. Therefore, it can easily realize the standardization of the home network system. The hardware and business management logic separation of the gateway improve the scalability of the system. The working mode and parameter configuration of the gateway can be set through a web browser. After the completion of the configuration, the system will directly enter the work mode properly, which can reduce the development workload of new home network applications. Experimental results show that the 
proposed gateway successfully realizes home network applications using little infrastructure, and it provides a faster and more flexible approach for building and deploying home automation networks.

\section{Conflict of Interests}

The authors declared that they have no conflict of interests regarding this work.

\section{Acknowledgments}

This work is partially supported by National Major Project (Grant no. 2010ZX03006-006), National 863 Program (Grant no. 2014AA01A702), National Natural Science Foundation Project of China (Grant nos. 61272379 and 61325018), Jiangsu Provincial Key Technology R\&D Program (Grant no. BE2012165), and the Ministry of Education Science and Technology Innovation Engineering Major Cultivation Project of China (Grant no. 107053). The authors would like to thank Ke-fa Wang and Kai-jian Yin for help with the experiments.

\section{References}

[1] A. J. Jara, M. A. Zamora-Izquierdo, and A. F. Skarmeta, "Interconnection framework for mHealth and remote monitoring based on the internet of things," IEEE Journal on Selected Areas in Communications, vol. 31, no. 9, pp. 47-65, 2013.

[2] K. Malhi, S. C. Mukhopadhyay, J. Schnepper, M. Haefke, and H. Ewald, "A zigbee-based wearable physiological parameters monitoring system," IEEE Sensors Journal, vol. 12, no. 3, pp. 423430, 2012.

[3] E. S. Nadimi, H. T. Søgaard, T. Bak, and F. W. Oudshoorn, "ZigBee-based wireless sensor networks for monitoring animal presence and pasture time in a strip of new grass," Computers and Electronics in Agriculture, vol. 61, no. 2, pp. 79-87, 2008.

[4] W.-W. Chang, T.-J. Sung, H.-W. Huang et al., "A smart medication system using wireless sensor network technologies," Sensors and Actuators A: Physical, vol. 172, no. 1, pp. 315-321, 2011.

[5] S. D. T. Kelly, N. K. Suryadevara, and S. C. Mukhopadhyay, "Towards the implementation of IoT for environmental condition monitoring in homes," IEEE Sensors Journal, vol. 13, no. 10, pp. 3846-3853, 2013.

[6] Q. Hu, F. Li, and C.-F. Chen, "A smart home test bed for undergraduate education to bridge the curriculum gap from traditional power systems to modernized smart grids," IEEE Transactions on Education, vol. 58, no. 1, pp. 32-38, 2015.

[7] M. Spadacini, S. Savazzi, and M. Nicoli, "Wireless home automation networks for indoor surveillance: technologies and experiments Internet of Things for wireless and mobile communication," EURASIP Journal on Wireless Communications and Networking, vol. 2014, no. 1, p. 6, 2014.

[8] S. K. Korkua and K. Thinsurt, "Design of ZigBee based WSN for smart demand responsive home energy management system," in Proceedings of the 13th International Symposium on Communications and Information Technologies (ISCIT '13), pp. 549-554, September 2013.

[9] D. S. Kim, S.-Y. Son, and J. Lee, "Developments of the in-home display systems for residential energy monitoring," IEEE Transactions on Consumer Electronics, vol. 59, no. 3, pp. 492-498, 2013.
[10] H. Y. Tung, K. F. Tsang, H. C. Tung, K. T. Chui, and H. R. Chi, "The design of dual radio ZigBee homecare gateway for remote patient monitoring," IEEE Transactions on Consumer Electronics, vol. 59, no. 4, pp. 756-764, 2013.

[11] I.-K. Hwang, D.-S. Lee, and J.-W. Baek, "Home network configuring scheme for all electric appliances using ZigBee-based integrated remote controller," IEEE Transactions on Consumer Electronics, vol. 55, no. 3, pp. 1300-1307, 2009.

[12] Y.-G. Ha, "Dynamic integration of zigbee home networks into home gateways using OSGI service registry," IEEE Transactions on Consumer Electronics, vol. 55, no. 2, pp. 470-476, 2009.

[13] G. Lopez, P. Moura, J. I. Moreno, and A. De Almeida, "ENERsip: M2M-based platform to enable energy efficiency within energypositive neighbourhoods," in Proceedings of the IEEE Conference on Computer Communications Workshops (INFOCOM WKSHPS '11), pp. 217-222, IEEE, Shanghai, China, April 2011.

[14] J. Swetina, G. Lu, P. Jacobs, F. Ennesser, and J. S. Song, "Toward a standardized common M2M service layer platform: introduction to oneM2M," IEEE Wireless Communications, vol. 21, no. 3, pp. 20-26, 2014.

[15] L. Foschini, T. Taleb, A. Corradi, and D. Bottazzi, "M2M-based metropolitan platform for IMS-enabled road traffic management in IoT,' IEEE Communications Magazine, vol. 49, no. 11, pp. 50-57, 2011.

[16] S. Adibi, "Link technologies and BlackBerry mobile health (mHealth) solutions: a review," IEEE Transactions on Information Technology in Biomedicine, vol. 16, no. 4, pp. 586-597, 2012.

[17] M. Chen, J. F. Wan, S. Gonzalez, X. F. Liao, and V. C. M. Leung, "A survey of recent developments in home M2M networks," IEEE Communications Surveys and Tutorials, vol. 16, no. 1, pp. 98-114, 2014. 


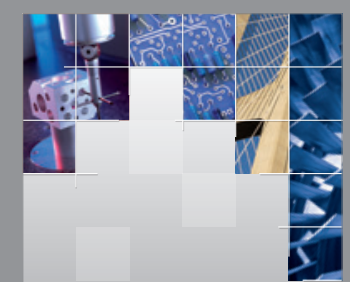

\section{Enfincering}
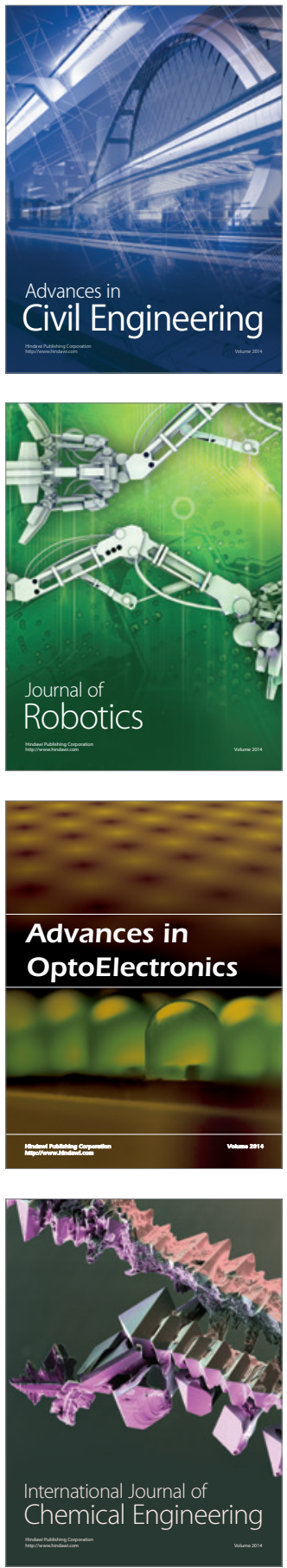

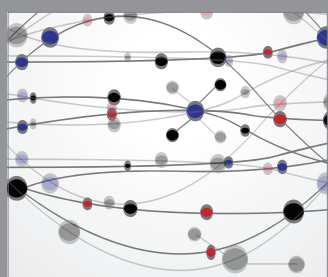

The Scientific World Journal

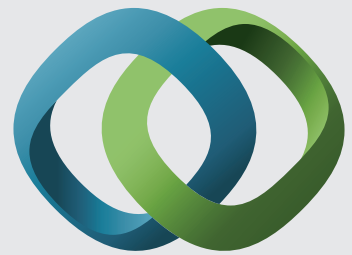

\section{Hindawi}

Submit your manuscripts at

http://www.hindawi.com
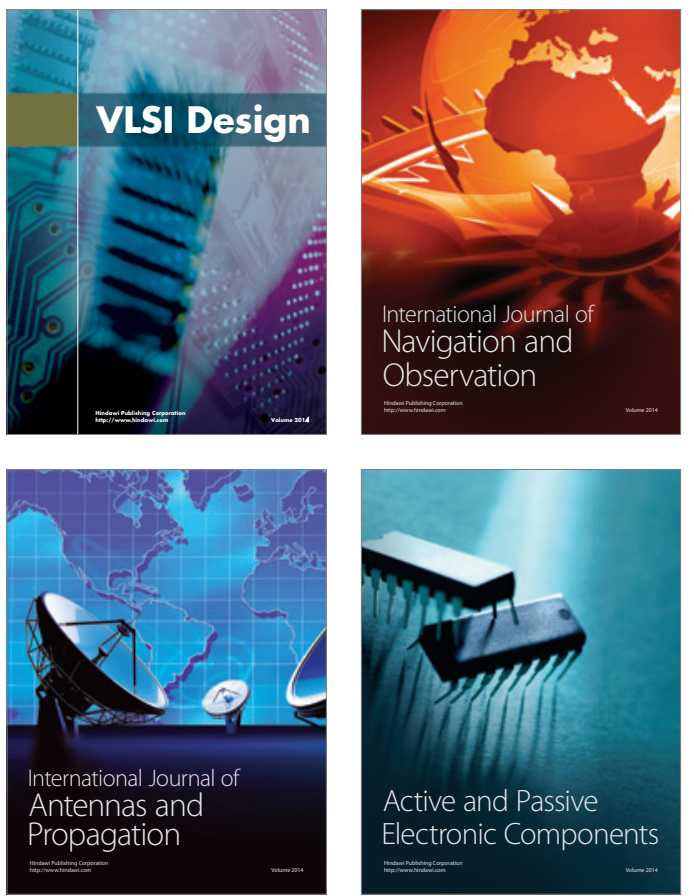
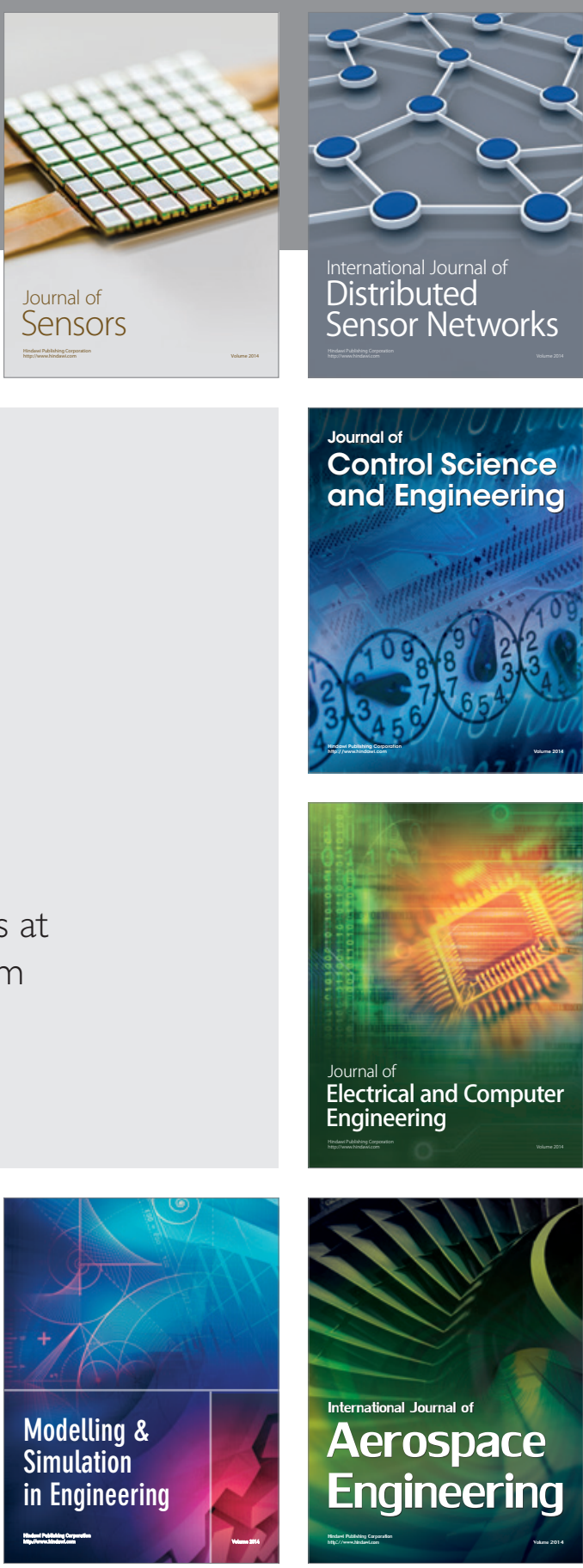

International Journal of

Distributed

Sensor Networks

Journal of

Control Science

and Engineering
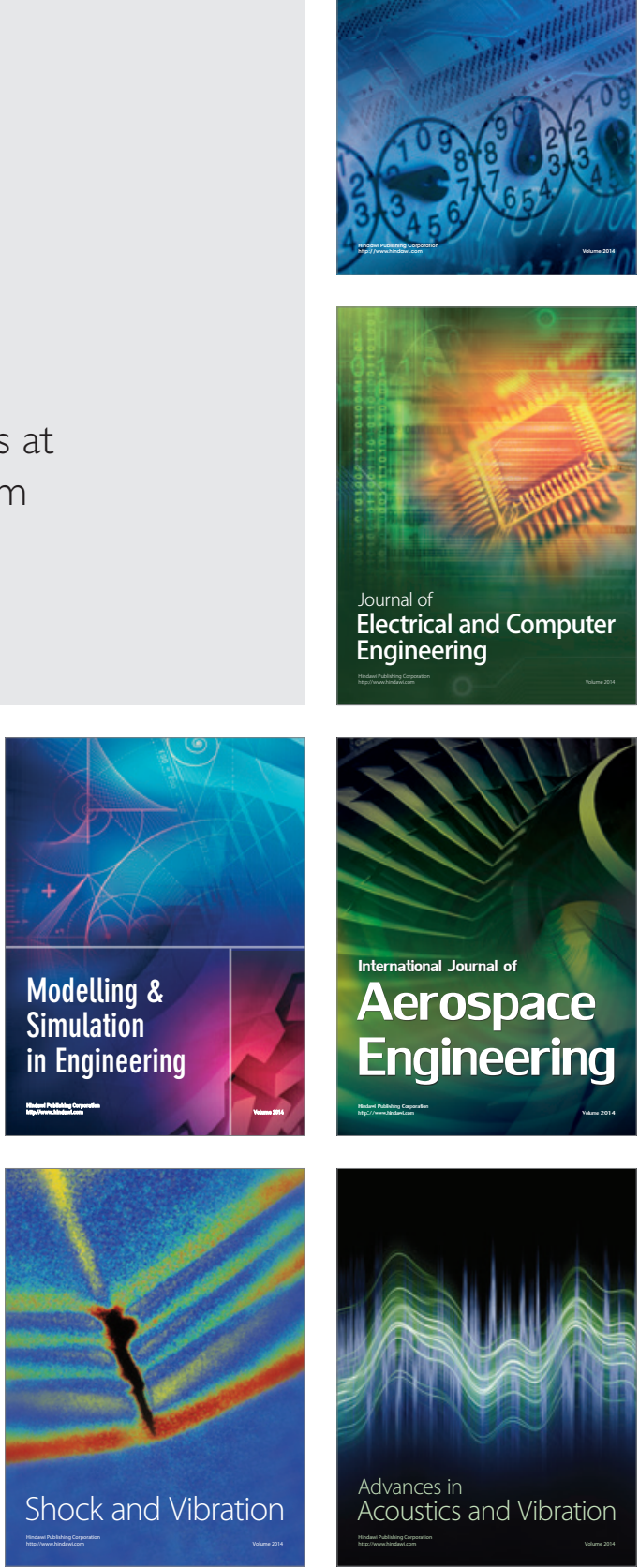